ESAIM: PROCEEDINGS, September 2012, Vol. 37, p. 117-135

E. Cancès and S. Labbé, Editors

\title{
WALL LAWS FOR VISCOUS FLUIDS NEAR ROUGH SURFACES
}

\author{
Dorin Bucur ${ }^{1}$, Anne-Laure Dalibard ${ }^{2}$ And David GÉrard-VAret ${ }^{3}$
}

\begin{abstract}
In this paper, we review recent results on wall laws for viscous fluids near rough surfaces, of small amplitude and wavelength $\epsilon$. When the surface is "genuinely rough", the wall law at first order is the Dirichlet wall law: the fluid satisfies a "no-slip" boundary condition on the homogenized surface. We compare the various mathematical characterizations of genuine roughness, and the corresponding homogenization results. At the next order, under ergodicity properties of the roughness distribution, a Navier wall law with a slip length of order $\varepsilon$ can be derived, that leads to better error estimates.

We also discuss the relationship beween the slip length and the position of the homogenized surface. In particular, we prove that for adherent rough walls, the Navier wall law associated to the roughness does not correspond to any tangible slip.
\end{abstract}

\section{INTRODUCTION}

Most works on Newtonian liquids assume the validity of the no-slip boundary condition: the velocity field of the liquid at a solid surface equals the velocity field of the surface itself. This assumption relies on both theoretical and experimental studies, carried over more than a century.

Still, with the recent surge of activity around microfluidics, the question of fluid-solid interaction has been reconsidered, and the consensus around the no-slip condition has been questioned. Several experimentalists, observing for instance water over mica, have reported significant slip. More generally, it has been claimed that, in many cases, the liquid velocity field $u$ obeys a Navier condition at the solid boundary $\Sigma$ :

$$
\left.\left(I_{d}-\nu \otimes \nu\right) u\right|_{\Sigma}=\left.\lambda\left(I_{d}-\nu \otimes \nu\right) D(u) \nu\right|_{\Sigma},\left.\quad u \cdot \nu\right|_{\Sigma}=0, \quad \lambda>0
$$

where $\nu$ is an inward normal vector to $\Sigma$, and $D(u)$ is the symmetric part of the gradient. Slip lengths $\lambda$ up to a few micrometers have been measured. This is far more than the molecular scale, and would therefore invalidate the (macroscopic) no-slip condition

$$
\left.u\right|_{\Sigma}=0 .
$$

Nevertheless, such experimental results are widely debated. For similar experimental settings, there are huge discrepancies between the measured values of $\lambda$. We refer to the article [23] for an overview.

In this debate around boundary conditions, the irregularity of the solid surface is a major issue. Again, its effect is a topic of intense discussion. On the one hand, some people argue that it increases the surface of friction, and may cause a decrease of the slip. On the other hand, it may

${ }^{1}$ Laboratoire de Mathématiques, Université de Savoie, Campus Scientifique, 73376 Le-Bourget-Du-Lac, France

2 DMA/CNRS, Ecole Normale Supérieure, 45 rue d'Ulm, 75005 Paris, France

${ }^{3}$ IMJ, 175 rue du Chevaleret, 75013 Paris, France

(C) EDP Sciences, SMAI 2012 


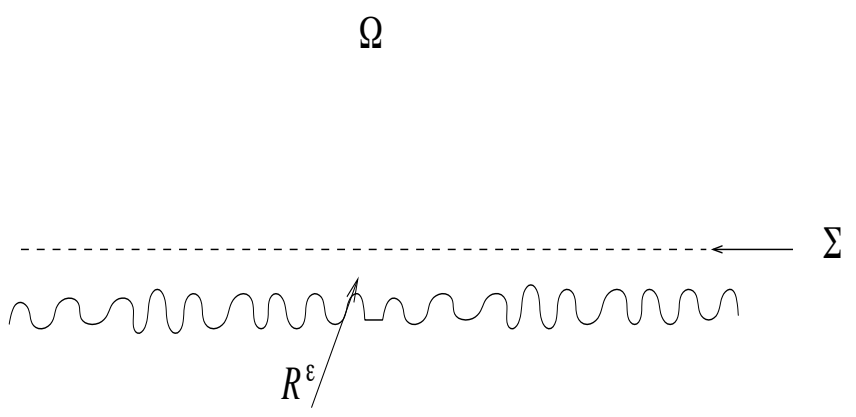

Figure 1. The rough domain $\Omega^{\varepsilon}$.

generate small scale phenomena favourable to slip. For instance, some rough hydrophobic surfaces seem more slippery due to the trapping of air bubbles in the humps of the roughness. Moreover, irregularity creates a boundary layer in its vicinity, meaning high velocity gradients. Thus, even though (Di) is satisfied at the rough boundary, there may be significant velocities right above. In other words, the no-slip condition may hold at the small scale of the boundary layer but not at the large scale of the mean flow. This phenomenon, due to scale separation, is called apparent slip in the physics litterature.

In parallel to experimental works, several theoretical studies have been carried, so as to clarify the role of roughness. Many of them relate to homogenization theory. First, the irregularity is modeled by small-scale variations of the boundary. Then, an asymptotic analysis is performed, as the small scales go to zero. The idea is to replace the constitutive boundary condition at the rough surface by a homogenized or effective boundary condition at the smoothened surface. In this way, one can describe the averaged effect of the roughness. We stress that such homogenized conditions (often called wall laws) are also of practical interest in numerical codes. They allow to filter out the small scales of the boundary, which have a high computational cost.

Let us now describe the setting of our study. We consider a two-dimensional rough channel

$$
\Omega^{\varepsilon}:=\Omega \cup \Sigma \cup R^{\varepsilon}
$$

where $\Omega=\mathbb{R} \times(0,1)$ is the smooth part, $R^{\varepsilon}$ is the rough part, and $\Sigma=\mathbb{R} \times\{0\}$ their interface. We assume that the rough part has typical size $\varepsilon$, that is

$$
R^{\varepsilon}:=\varepsilon R, \quad R:=\left\{y, 0>y_{2}>\omega\left(y_{1}\right)\right\}
$$

for a Lipschitz function $\omega: \mathbb{R} \mapsto(-1,0)$. We also introduce

$$
\Gamma^{\varepsilon}:=\varepsilon \Gamma, \quad \Gamma:=\left\{y, y_{2}=\omega\left(y_{1}\right)\right\}
$$

See Figure 1 for notation. We consider in this channel a steady flow $u^{\varepsilon}$. It is modeled by the stationary Navier-Stokes system, with a prescribed flux $\phi$ across a vertical cross-section $\sigma^{\varepsilon}$ of $\Omega^{\varepsilon}$. Moreover, to cover all interesting cases, we shall consider either pure slip, partial slip or no-slip at the rough boundary $\Gamma^{\varepsilon}$. This means that the constant $\lambda^{\varepsilon}$ below shall be either $+\infty$, positive or 
zero. For simplicity, we assume no-slip at the upper boundary. We get eventually

$$
\left\{\begin{array}{c}
-\Delta u^{\varepsilon}+u^{\varepsilon} \cdot \nabla u^{\varepsilon}+\nabla p^{\varepsilon}=0, x \in \Omega^{\varepsilon}, \\
\operatorname{div} u^{\varepsilon}=0, x \in \Omega^{\varepsilon}, \\
\left.u^{\varepsilon}\right|_{x_{2}=1}=0, \int_{\sigma^{\varepsilon}} u_{1}^{\varepsilon}=\phi, \\
\left.\left(I_{d}-\nu \otimes \nu\right) u^{\varepsilon}\right|_{\Gamma^{\varepsilon}}=\left.\lambda^{\varepsilon}\left(I_{d}-\nu \otimes \nu\right) D\left(u^{\varepsilon}\right) \nu\right|_{\Gamma^{\varepsilon}},\left.u^{\varepsilon} \cdot \nu\right|_{\Gamma^{\varepsilon}}=0 .
\end{array}\right.
$$

Notice that the flux integral in the third equation does not depend on the location of the crosssection $\sigma^{\varepsilon}$, thanks to the divergence-free and impermeability conditions. We also emphasize that this problem has a singularity in $\varepsilon$, due to the high frequency oscillation of the boundary. Thus, the problem is to replace the singular problem in $\Omega^{\varepsilon}$ by a regular problem in $\Omega$. The idea is to keep the same Navier-Stokes equations

$$
\left\{\begin{aligned}
-\Delta u+u \cdot \nabla u+\nabla p & =0, x \in \Omega \\
\operatorname{div} u & =0, x \in \Omega \\
\left.u\right|_{x_{2}=1}=0, \quad \int_{\sigma} u_{1} & =\phi \quad \text { where } \sigma=(0,1),
\end{aligned}\right.
$$

but with a boundary condition at the artificial boundary $\Sigma$ which is regular in $\varepsilon$. The problem is to find the most accurate condition.

A series of papers has addressed this problem, starting from the standard Dirichlet condition at $\Gamma^{\varepsilon}\left(\lambda^{\varepsilon}=0\right.$ in $\left.\left(\mathrm{NS}^{\varepsilon}\right)\right)$. Before stating the results, let us introduce the functional spaces which will be central in our study. Notice that the domain $\Omega$ is unbounded in the horizontal direction, but we wish to allow velocity fields such as the Poiseuille flow to belong to our functional space. Therefore a standard choice is to work with

$$
\begin{gathered}
L_{u l o c}^{2}(\Omega)=\left\{u \in L_{l o c}^{2}(\Omega), \sup _{x_{1} \in \mathbb{R}} \int_{\left(x_{1}, x_{1}+1\right) \times(0,1)}|u|^{2}<\infty\right\}, \\
H_{u l o c}^{1}(\Omega)=\left\{u \in H_{l o c}^{1}(\Omega), \sup _{x_{1} \in \mathbb{R}}\|u\|_{H^{1}\left(\left(x_{1}, x_{1}+1\right) \times(0,1)\right)}<\infty\right\},
\end{gathered}
$$

equipped with the norms

$$
\|u\|_{L_{u l o c}^{2}}=\sup _{x_{1} \in \mathbb{R}}\|u\|_{L^{2}\left(\left(x_{1}, x_{1}+1\right) \times(0,1)\right)}, \quad\|u\|_{H_{u l o c}^{1}}=\sup _{x_{1} \in \mathbb{R}}\|u\|_{H^{1}\left(\left(x_{1}, x_{1}+1\right) \times(0,1)\right)} .
$$

Loosely, two main facts have been established:

(1) For any roughness profile $\omega$, the Dirichlet condition (Di) provides a $O(\varepsilon)$ approximation of $u^{\varepsilon}$ in $L_{u l o c}^{2}(\Omega)$.

(2) For generic roughness profile $\omega$, the Navier condition does better, choosing $\lambda=\alpha \varepsilon$ for some good positive constant $\alpha$ in $(\mathrm{Na})$.

Of course, such statements are only the crude translations of cumulative rigorous results. Up to our knowledge, the pioneering results on wall laws are due to Achdou, Pironneau and Valentin [1,3], and Jäger and Mikelic [19,20], who considered periodic roughness profiles $\omega$. See also [4] on this periodic case. The extension to arbitrary roughness profiles has been studied by the third author (and coauthors) in articles $[5,16,17]$. The expression generic roughness profile means functions $\omega$ with ergodicity properties (for instance, $\omega$ is random stationary, or almost periodic). We refer to the forementioned works for all details and rigorous statements. Let us just mention that the slip length $\alpha \varepsilon$ is related to a boundary layer of amplitude $\varepsilon$ near the rough boundary. It is the mathematical expression of the apparent slip discussed earlier.

Beyond the special case $\lambda^{\varepsilon}=0$, some studies have dealt with the general case $\lambda^{\varepsilon} \in[0,+\infty]$. The limit $u^{0}$ of $u^{\varepsilon}$, and the condition that it satisfies at $\Sigma$ have been investigated. In brief, the 
striking conclusion of these studies is that, as soon as the boundary is genuinely rough, $u^{0}$ satisfies a no-slip condition at $\Sigma$. This idea has been developped in [11] for a periodic roughness pattern $\omega$. It has been generalized to arbitrary roughness pattern by the first author and coauthors in [8]. In this last article, the assumption of genuine roughness is expressed in terms of Young measures. Eventually, error estimates are derived by the last two authors in [12]; the roughness assumption takes there the form of a Poincaré inequality in the domain $R$. We will give precise statements in section 2 .

These results can be seen as a mathematical justification of the no-slip condition. Indeed, any realistic boundary is rough. If one is only interested in scales greater than the scale $\varepsilon$ of the roughness, then $(\mathrm{Di})$ is an appropriate boundary condition, whatever the microscopic phenomena behind. Still, as in the case $\lambda^{\varepsilon}=0$, one may be interested in more quantitative estimates. How good is the boundary condition? Can it be improved? Is there possibility of a $O(\varepsilon)$ slip? Such questions are especially important in microfluidics, a domain in which minimizing wall friction is crucial (see [26]). They were tackled in [12], where the last two authors proved the existence of a better homogenized boundary condition, in a stationary random setting, and under the assumption that a Korn-type inequality holds in the domain $R$.

Once the existence of a slip length $\alpha \varepsilon$ is known, the natural question is to investigate the incidence of the geometry of $\Gamma$ on the coefficient $\alpha$, and more precisely to optimize the geometry in order to achieve its maximal value. This is the main novelty of this paper: starting from a Dirichlet condition in a periodic setting $\left(\lambda^{\varepsilon}=0\right)$, we prove that the maximal value is achieved for a flat boundary. The precise result is the following (below and in the rest of the article, $\langle f\rangle$ denotes the mean value of a periodic function $f$ ):

Theorem 1. Assume that $\lambda^{\varepsilon}=0$, and that $\omega$ is periodic, with $\langle\omega\rangle=-\frac{1}{2}$.

Then the slip coefficient $\alpha$ is maximal when $\omega \equiv-\frac{1}{2}$.

This theorem is somehow negative. It expresses that in the context of hydrophilic surfaces (for which a microscopic Dirichlet condition holds), the roughness can not generate any tangible slip. In other words, the positivity of the coefficient $\alpha$ is misleading: it just relies on the position of the homogenized surface, which in our model lies above the roughness boundary. For a given volume of the channel (encoded by the constraint $\langle\omega\rangle=-\frac{1}{2}$ ) and a given flux, that is for a given input of energy, the flat boundary minimizes the energy loss due to friction.

Let us now conclude this introduction with a few words about some possible extensions of the results presented here. First, let us emphasize that in the periodic setting, all the results quoted in this paper which hold in two dimensions (Dirichlet wall law at first order, Navier wall law at the second order) remain true in three dimensions. In fact, in the paper [11], the authors work in a three dimensional setting. The construction of boundary layers in the neighbourhood of a periodic wall is also straightforward, and the dimension of the space is irrelevant in this context. When $\omega$ is not periodic, the Dirichlet wall law at first order still holds in three dimensions when the wall is genuinely rough (we refer to the next section for precise definitions). More generally, all the proofs relying mainly on weak convergence, and therefore on a priori estimates on $u^{\varepsilon}$, are not affected by the space dimension. However, the derivation of error estimates and the construction of boundary layers are much more involved, and go beyond the scope of this paper.

We also mention that most results could probably be extended without difficulty to a time dependent setting, or to a geometry where the wall is not flat at first order. Such generalizations are in fact considered respectively in $[8,11]$, and show therefore the validity of the Dirichlet wall law at first order in a very general context. The simple setting chosen in this paper allows us to identify explicitly the main flow, which is a Poiseuille flow. But it seems very likely that the construction of boundary layers, for instance, could be performed even if the main flow is not known; we refer for instance to $[13,18]$ for the construction of boundary layers near non-flat boundaries.

The organization of the paper is the following. The next two sections are dedicated to a review of previous results: section 2 is concerned with the Dirichlet wall law, with a comparison of the different assumptions on the roughness appearing in the literature, and section 3 with the Navier wall law and the existence of a boundary layer. Eventually, Theorem 1 is proved in section 4 . 


\section{Dirichlet WAll LAW AT First ORDER}

\subsection{A short review of "weak" convergence results}

The idea behind the Dirichlet wall law is the following: since the flow is tangent to the rough boundary $\Gamma^{\varepsilon}$, the solution of $\left(\mathrm{NS}^{\varepsilon}\right)$ converges towards a Poiseuille flow, provided the boundary is sufficiently rough. The no-slip wall law is obtained simply by passing to the limit in the boundary condition

$$
\left.u^{\varepsilon} \cdot \nu\right|_{\Gamma^{\varepsilon}}=0 .
$$

The arguments rely on weak convergence techniques (in $H^{1}$ ), belonging to $\Gamma$-convergence theory, rather than energy estimates.

The pioneering work in this direction is the paper by Casado-Diaz, Fernandez-Cara and Simon [11]. Their result, modulo a slight rephrasing to match the present setting, is the following:

Proposition 2 (Casado-Diaz et al.). Assume that $\omega \in \mathcal{C}^{1}(\mathbb{R})$ is periodic and is not constant.

Let $v^{\varepsilon} \in H_{u l o c}^{1}\left(\Omega^{\varepsilon}\right)$ such that

$$
\begin{array}{r}
\sup _{\varepsilon>0}\left\|v^{\varepsilon}\right\|_{H_{u l o c}^{1}\left(\Omega^{\varepsilon}\right)}<\infty, \\
v^{\varepsilon} \cdot \nu_{\mid \Gamma^{\varepsilon}}=0 .
\end{array}
$$

Let $v^{0}$ be the weak limit in $w-H_{u l o c}^{1}$ of $v^{\varepsilon}$ along any subsequence. Then

$$
v_{\mid \Sigma}^{0}=0
$$

Remark 3. - Actually, the result of Casado-Diaz, Fernandez-Cara and Simon is established in a 3-dimensional setting, i.e. for a function $\omega \in \mathcal{C}^{1}\left(\mathbb{R}^{2}\right)$. In this case, the "nondegeneracy assumption" on $\omega$ reads

$$
\forall y_{h} \in \mathbb{R}^{2} \backslash\{0\}, \quad \exists z_{h} \in \mathbb{R}^{2}, \exists c \in \mathbb{R}, \quad \omega\left(z_{h}+c y_{h}\right) \neq \omega\left(z_{h}\right),
$$

meaning that $\omega$ has no invariant direction.

- The Dirichlet wall law for system $\left(\mathrm{NS}^{\varepsilon}\right)$ is therefore a mere consequence of Proposition 2. Indeed, in the periodic case, it can be easily proved, thanks to energy estimates, that the solution $u^{\varepsilon}$ of $\left(\mathrm{NS}^{\varepsilon}\right)$ is uniformly bounded in $H_{u l o c}^{1}$.

The heuristics of the proof goes as follows: if $v^{\varepsilon}$ is bounded in $H^{1}$, then the trace of $v^{\varepsilon}$ on $\Sigma$ converges strongly in $L^{2}$ (up to a subsequence) towards $\left.v^{0}\right|_{\Sigma}$, so that

$$
\left.\left.v^{0}\right|_{\Sigma} \cdot \nu\right|_{\Gamma^{\varepsilon}}=o(1)
$$

Decoupling the macroscopic variable $x$ and the microscopic variable $y_{1}$, which describes the asperities of $\Gamma$, we obtain

$$
\left.\left.v^{0}\right|_{x_{2}=0} \cdot \nu\left(y_{1}\right)\right|_{\Gamma}=0
$$

Hence, as long as $\nu\left(y_{1}\right)$ takes at least two different values, $v^{0}$ vanishes on the lower boundary.

This result was then extended to arbitrary roughness patterns by Bucur, Feireisl, Necasova and Wolf in [8]. In this article, the assumption of genuine roughness is expressed in terms of Young measures. When recast in our 2D setting, it reads:

(H) The family of Young measures $\left(d \mu_{y_{1}}\right)_{y_{1} \in \mathbb{R}}$ associated with the sequence $\left(\omega^{\prime}(\cdot / \varepsilon)\right)_{\varepsilon}$ is s.t.

$$
d \mu_{y_{1}} \neq \delta_{0} \text { (the Dirac mass at zero), for almost every } y_{1} \in \mathbb{R} \text {. }
$$

Under $(\mathrm{H})$, one can show that $u^{\varepsilon}$ locally converges in $H^{1}$-weak to the famous Poiseuille flow:

$$
u^{0}(x)=\left(U^{0}\left(x_{2}\right), 0\right), \quad U^{0}\left(x_{2}\right)=6 \phi x_{2}\left(1-x_{2}\right)
$$


which is solution of (NS)-(Di). We refer to [8] for all details.

If the non-degeneracy assumption on the roughness is relaxed, then the flow may no longer satisfy a Dirichlet boundary condition at the limit, as the (three-dimensional) examples in $[9,10]$ show: these articles study the asymptotic behaviour of a fluid slipping on a rough boundary made of ribblets (i.e. $\omega$ is invariant in one direction, and "genuinely rough" in the other). The authors then prove the validity of the Navier condition at the limit, with a slip length of order one.

Let us emphasize that the results of [11] and [8] are true for any sequence $\lambda^{\varepsilon}$, and in particular if $\lambda^{\varepsilon}=0$ (adherent boundary). However, in this case, the non-degeneracy assumptions on the roughness are irrelevant, and the no-slip condition always holds in the limit, although we are not aware of any weak convergence proof of this fact. Results giving an error estimate will be provided in the next paragraph.

\subsection{Rate of convergence}

Error estimates for the Dirichlet wall law in the present context were first derived in a periodic setting by Jäger and Mikelic in [19], in the case $\lambda^{\varepsilon}=0$. The authors prove that if $u^{0}$ is the Poiseuille flow in the channel $\Omega$, then

$$
\left\|u^{\varepsilon}-u^{0}\right\|_{L^{2}}=O(\varepsilon) .
$$

Let us emphasize that the work of Jäger and Mikelic takes place in a bounded channel (with given pressures at the entrance and the exit of the channel). In such a setting, the derivation of $L^{2}$ estimates starting from a Dirichlet boundary condition on $\Gamma^{\varepsilon}$ does not raise any technical difficulty: extending $u^{0}$ by zero below $\Sigma$, one infers that $v^{\varepsilon}=u^{\varepsilon}-u^{0}$ satisfies

$$
\left\{\begin{aligned}
-\Delta v^{\varepsilon}+\tilde{u}^{0} \cdot \nabla v^{\varepsilon}+v^{\varepsilon} \cdot \nabla \tilde{u}^{0}+v^{\varepsilon} \cdot \nabla v^{\varepsilon}+\nabla q & =f^{\varepsilon}, x \in \Omega^{\varepsilon}, \\
\operatorname{div} v^{\varepsilon} & =0, x \in \Omega^{\varepsilon}, \\
\left.v^{\varepsilon}\right|_{x_{2}=1}=0, \int_{\sigma^{\varepsilon}} v_{1}^{\varepsilon} & =0, \\
\left.v^{\varepsilon}\right|_{\Gamma^{\varepsilon}}=0 &
\end{aligned}\right.
$$

where $f^{\varepsilon}$ is a source term of order $\sqrt{\varepsilon}$ in $L^{2}$. This leads naturally to $O(\sqrt{\varepsilon})$ estimates in $H^{1}$. Using a rescaled Poincaré inequality in $R^{\varepsilon}$, the authors infer that

$$
\left\|\left.v^{\varepsilon}\right|_{\Sigma}\right\|_{L^{2}(\Sigma)}=O(\varepsilon), \quad\left\|v^{\varepsilon}\right\|_{L^{2}\left(R^{\varepsilon}\right)}=O\left(\varepsilon^{3 / 2}\right) .
$$

The $O(\varepsilon)$ estimate in $L^{2}$ then follows from classical results on the very weak solutions of the Stokes system (or Oseen's problem); the duality arguments leading to this estimate are recalled in [5].

These results were extended by Basson and the third author in [5], for an arbitrary profile $\omega$, still in the case $\lambda^{\varepsilon}=0$. The difficulty then relies on the derivation of $H_{u l o c}^{1}$ and $L_{u l o c}^{2}$ estimates, which require truncations in $x_{1}$, since the domain $\Omega^{\varepsilon}$ is unbounded in this direction. In fact, the well-posedness of the system $\left(\mathrm{NS}^{\varepsilon}\right)$ itself is not obvious.

In the case $\lambda^{\varepsilon}>0$, additional assumptions ensuring that $\omega$ is "genuinely rough" are necessary in order to ensure a Dirichlet wall law, in the spirit of assumption (H). In [12], the last two authors of the present paper derive error estimates for any sequence $\lambda^{\varepsilon}$ provided a Poincaré inequality holds in the rough part of $\Omega^{\varepsilon}$, namely

$\left(H^{\prime}\right)$ There exists $\mathcal{C}>0$, such that for all 2-D fields $u \in C_{c}^{\infty}(\bar{R})$ satisfying $\left.u \cdot \nu\right|_{\Gamma}=0$,

$$
\|u\|_{L^{2}(R)} \leq \mathcal{C}\|\nabla u\|_{L^{2}(R)} .
$$

Notice that this inequality is strongly related (almost identical, in fact) to the "uniform rugosity effect" in the recent paper by Bonnivard and the first auhor, [6]. 
The arguments leading to error estimates in [5] and [12] follow the work of Ladyzhenskaya and Solonnikov [22]. The idea is to prove by backward induction on $k$ that

$$
E_{k} \leq C_{0} \phi^{2}(k+1) \varepsilon, \quad C_{0} \text { large enough, }
$$

where

$$
E_{k}:=\|v\|_{L^{2}\left(\Omega_{k}^{\varepsilon}\right)}^{2}+\|\nabla v\|_{L^{2}\left(\Omega_{k}^{\varepsilon}\right)}^{2}+\|D(v)\|_{L^{2}\left(\Omega_{k}^{\varepsilon}\right)}^{2},
$$

where $\Omega_{k}^{\varepsilon}=\Omega^{\varepsilon} \cap\left\{\left|x_{1}\right| \leq k\right\}$. Once the bound on the $E_{k}$ 's is proved, using it with $k=1$ yields $E_{1} \leq C \phi^{2} \varepsilon$, and thus provides a $O(\sqrt{\varepsilon})$ bound in $H_{u l o c}^{1}$.

Let us explain briefly how (2.4) is derived. First, for $n \in \mathbb{N}$, an approximate solution of (2.3) in a truncated channel $\Omega_{n}^{\varepsilon}=\Omega^{\varepsilon} \cap\left\{\left|x_{1}\right| \leq n\right\}$ is constructed with $f^{\varepsilon}=1_{R^{\varepsilon}}(-12 \phi, 0)$ and with

$$
\left.v_{\tau}^{\varepsilon}\right|_{\Gamma^{\varepsilon}}=\left.\lambda^{\varepsilon}\left(D\left(v^{\varepsilon}\right) \nu\right)_{\tau}\right|_{\Gamma^{\varepsilon}},\left.\quad v^{\varepsilon} \cdot \nu\right|_{\Gamma^{\varepsilon}}=0
$$

as boundary condition on the rough surface, where $v_{\tau}$ denotes the tangential part of $v$. The system is supplemented with the following jump conditions at the interface $\Sigma$

$$
\left.\left[v^{\varepsilon}\right]\right|_{\Sigma}=0,\left.\quad\left[-D\left(v^{\varepsilon}\right) e_{2}+q e_{2}\right]\right|_{\Sigma}=(-6 \phi, 0)
$$

and with Dirichlet boundary conditions on the lateral boundaries $\left\{x_{1}= \pm n\right\}$. In order to lighten the notation, this approximate solution is denoted by $v$ in the rest of this paragraph.

Multiplying (2.3) formally by $v$, we obtain

$$
\int_{\Omega_{n}^{\varepsilon}}|D(v)|^{2}+\left(\lambda^{\varepsilon}\right)^{-1} \int_{\Gamma_{n}^{\varepsilon}}\left|v_{\tau}\right|^{2}=-\int_{\Omega_{n}^{\varepsilon}}\left(\tilde{u}^{0} \otimes v+v \otimes \tilde{u}^{0}\right): D(v)-\int_{R_{n}^{\varepsilon}} 12 \phi v_{1}+\int_{\Sigma_{n}^{\varepsilon}} 6 \phi v_{1} .
$$

As $\left\|\nabla u^{0}\right\|_{\infty} \leq C \phi$, we obtain

$$
\|D(v)\|_{L^{2}\left(\Omega_{n}^{\varepsilon}\right)}^{2} \leq C \phi\left(\|v\|_{L^{2}\left(\Omega_{n}^{\varepsilon}\right)}\|D(v)\|_{L^{2}\left(\Omega_{n}^{\varepsilon}\right)}+\sqrt{n \varepsilon}\|v\|_{L^{2}\left(R_{n}^{\varepsilon}\right)}+\sqrt{n}\|v\|_{L^{2}\left(\Sigma_{n}^{\varepsilon}\right)}\right) .
$$

As $v$ is zero at the upper boundary of the channel, Poincaré inequality applies, to provide

$$
\|v\|_{L^{2}\left(\Omega_{n}^{\varepsilon}\right)} \leq C\|\nabla v\|_{L^{2}\left(\Omega_{n}^{\varepsilon}\right)}
$$

where $C$ depends only on the height of the channel. A rescaled version of assumption (H') ensures that

$$
\begin{array}{r}
\|v\|_{L^{2}\left(R_{n}^{\varepsilon}\right)} \leq C \varepsilon\|\nabla v\|_{L^{2}\left(R_{n}^{\varepsilon}\right)}, \\
\|v\|_{L^{2}\left(\Sigma_{n}^{\varepsilon}\right)} \leq C^{\prime} \sqrt{\varepsilon}\|\nabla v\|_{L^{2}\left(R_{n}^{\varepsilon}\right)} .
\end{array}
$$

Eventually, using a homogeneous version of the Korn inequality (see $[15,24]$ ), we infer $E_{n} \leq$ $C\|D(v)\|_{L^{2}\left(\Omega_{n}^{\varepsilon}\right)}^{2}$, so that, for $\phi$ small enough, we have the global estimate

$$
E_{n} \leq C \phi^{2} n \varepsilon
$$

Then the idea is to prove by backward induction on $k$ that (2.4) holds uniformly in $n$. Inequality (2.8) is used as the induction assumption; to go from $k+1$ to $k$, a truncation in $x_{1}$ is used, together with energy estimates similar to the ones above.

\subsection{Comparison of the assumptions on the boundary}

When $\lambda^{\varepsilon}>0$, as we already mentioned, assumptions on the roughness of the boundary are necessary in order to retrieve a Dirichlet wall law. Assumption $(\mathrm{H})$ is sufficient for $\Gamma$-convergence techniques, while (H') ensures that error estimates hold. 
Let us now compare the two assumptions in the most common settings: it can be proved (see [12]) that if $\omega$ is Lipschitz, then

$$
\left(H^{\prime}\right) \Longleftrightarrow \exists A>0, \inf _{y_{1} \in \mathbb{R}} \int_{0}^{A}\left|\omega^{\prime}\left(y_{1}+t\right)\right|^{2} d t>0 .
$$

Therefore, the following facts are easy to check [12]:

- If $\omega$ is periodic, or quasi-periodic and smooth $\left(\omega \in \mathcal{C}^{2}(\mathbb{R})\right)$, then $\left(\mathrm{H}^{\prime}\right)$ holds if and only if $\omega$ is not constant.

- Assume now that $\omega$ is random and stationary in an ergodic setting. Denote by $(M, \mu)$ the underlying probability space, and by $\left(\tau_{y_{1}}\right)_{y_{1} \in \mathbb{R}}$ the measure-preserving transformation group acting on $M$. We recall that there exists a function $F \in L^{\infty}(M)$ such that

$$
\omega\left(y_{1}, m\right)=F\left(\tau_{y_{1}} m\right), \quad y_{1} \in \mathbb{R}, m \in M .
$$

As in [5], we define the stochastic derivative of $F$ by

$$
\partial_{m} F(m):=\omega^{\prime}(0, m) \quad \forall m \in M,
$$

so that $\omega^{\prime}\left(y_{1}, m\right)=\partial_{m} F\left(\tau_{y_{1}} m\right)$ for $\left(y_{1}, m\right) \in \mathbb{R} \times M$.

Then

$$
\left(H^{\prime}\right) \Longleftrightarrow \exists A>0, \operatorname{essinf}_{m \in M} \int_{0}^{A}\left|\partial_{m} F\left(\tau_{t} m\right)\right|^{2} d t>0 .
$$

In general, the above assumption is more stringent than merely requiring that $F$ is not constant almost surely.

On the other hand, in the stationary ergodic case, the family of Young measures associated with the sequence $\omega^{\prime}(\cdot / \varepsilon)$ can be easily identified: indeed, according to the results of Bourgeat, Mikelic and Wright (see [7]), for all $G \in \mathcal{C}^{1}(\mathbb{R})$ and for all test function $\varphi \in L^{1}(\mathbb{R} \times M)$, there holds

$$
\int_{\mathbb{R} \times M} G\left(\omega^{\prime}\left(\frac{y_{1}}{\varepsilon}, m\right)\right) \varphi\left(y_{1}, m\right) d y_{1} d \mu(m) \rightarrow \int_{\mathbb{R} \times M} E\left[G\left(\partial_{m} F\right)\right] \varphi\left(y_{1}, m\right) d y_{1} d \mu(m) .
$$

By definition of the Young measure, the left-hand side also converges (up to a subsequence) towards

$$
\left.\int_{\mathbb{R} \times M}\left\langle G, d \mu_{y_{1}}\right\rangle \varphi\left(y_{1}, m\right)\right) d y_{1} d \mu(m) .
$$

As a consequence, we obtain

$$
\left\langle G, d \mu_{y_{1}}\right\rangle=E\left[G\left(\partial_{m} F\right)\right] \quad \text { for a.e. } y_{1} \in \mathbb{R} .
$$

Hence condition $(\mathrm{H})$ is equivalent (in the stationary ergodic setting) to

$$
E\left[\left|\partial_{m} F\right|^{2}\right]>0, \quad \text { i.e. Fnon constant a.s. }
$$

We deduce that assumptions $(\mathrm{H})$ and $\left(\mathrm{H}^{\prime}\right)$ are equivalent in the periodic and quasi-periodic settings. In the general stationary ergodic setting, however, condition (2.9) is stronger than $(\mathrm{H})$, but seems necessary in order to have quantitative error estimates.

\section{NAVIER WALL LAW AT SECOND ORDER}

In order to improve our description of $u^{\varepsilon}$, we must analyze the behaviour of the fluid in the boundary layer. The starting point of this analysis is a formal expansion: we anticipate that, near the rough boundary, we have

$$
u^{\varepsilon}(x)=u^{0}(x)+6 \phi \varepsilon v(x / \varepsilon)
$$


where $u^{0}$ is the Poiseuille flow, and $v=v(y)$ is a boundary layer corrector, due to the fact that $\left.u^{0}\right|_{\Gamma^{\varepsilon}}$ does not satisfy either the Dirichlet boundary condition when $\lambda^{\varepsilon}=0$, or the slip boundary condition when $\lambda^{\varepsilon} \neq 0$.

Classically, the rescaled variable $y$ belongs to the bumped half plane $\Omega^{b l}:=\left\{y, y_{2}>\omega\left(y_{1}\right)\right\}$, and by plugging the expansion in $\left(\mathrm{NS}^{\varepsilon}\right)$, one finds that

$$
\left\{\begin{array}{rlrl}
-\Delta v+\nabla p & =0, & & y \in \Omega^{b l} \\
\operatorname{div} v & =0, & y \in \Omega^{b l}
\end{array}\right.
$$

The boundary condition on $\partial \Omega^{b l}$ then depends on the scaling of $\lambda^{\varepsilon}$. In this paragraph, we will review mainly two cases:

- $\lambda^{\varepsilon}=0$ for all $\varepsilon>0$ (adherent boundary): in this case, the boundary condition on $\partial \Omega^{b l}$ is an inhomogeneous Dirichlet boundary condition, namely

$$
\left.v\right|_{\Gamma}=-\left(y_{2}, 0\right)
$$

- $\inf _{\varepsilon>0} \lambda^{\varepsilon}>0$ (slippery boundary): in this case, the boundary condition on $\partial \Omega^{b l}$ is an inhomogeneous Navier boundary condition, namely

$$
\begin{aligned}
(D(v) \nu)_{\tau}=-\left(D\left(\left(y_{2}, 0\right)\right) \nu\right)_{\tau}, & y \in \partial \Omega^{b l} \\
v \cdot \nu=-\left(y_{2}, 0\right) \cdot \nu, & y \in \partial \Omega^{b l}
\end{aligned}
$$

where

$$
\nu=\nu(y):=\frac{1}{\sqrt{1+\omega^{\prime 2}\left(y_{1}\right)}}\left(-\omega^{\prime}\left(y_{1}\right), 1\right)
$$

is a unit normal vector.

In all cases, the inhomogeneous boundary terms come from the Poiseuille flow $\left(x_{2}\left(1-x_{2}\right) \approx \varepsilon y_{2}\right.$ near the boundary).

\subsection{Well-posedness results for the boundary layer system}

The first result of well-posedness for (BL)-(BL-Di) was proved by Achdou et al. in [1] (see also $[2,3]$ ) for periodic roughness. These results were then refined by Jäger and Mikelic in [19] (see also [20]) and by Amirat et al. [4]. If the function $\omega$ is periodic, say with period $L$, the functional setting for $(\mathrm{BL})$ is clear: one looks for a solution in the space

$$
\left\{v \in L_{l o c}^{2}\left(\Omega^{b l}\right), v \text { is } L-\text { periodic in } y_{1}, \int_{0}^{L} \int_{\omega\left(y_{1}\right)}^{\infty}|\nabla v|^{2} d y_{2} d y_{1}<\infty\right\} .
$$

The well-posedness relies on a mere application of the Lax-Milgram Lemma.

If the roughness is not periodic, on the other hand, the boundary layer domain becomes unbounded in all directions, which prevents the use of functional inequalities such as the Poincaré inequality. The construction of boundary layers in a random and stationary environment for system (BL)-(BL-Di) was achieved by Basson and the third author in [5] (see also [16]). However, the method of [5] relies on the use of the random environment; the boundary layer term is not constructed for any realization of the rough boundary, but is intrinsically probabilistic.

This difficulty was overcome by the third author and Masmoudi in [17] for the boundary condition (BL-Di). The two main steps of the construction are the following:

(1) One replaces system (BL) by an equivalent system, set in the channel

$$
\Omega^{b l,-}:=\Omega^{b l} \cap\left\{y_{2}<0\right\} .
$$

This equivalent system involves a nonlocal boundary condition at $y_{2}=0$, with a Dirichletto-Neumann type operator. 
(2) Once brought back to the channel $\Omega^{b l,-}$, one can derive energy estimates and follow the same general strategy as in the previous section, based on the truncated energies

$$
E_{k}:=\|v\|_{L^{2}\left(\Omega_{k}^{b l,-}\right)}^{2}+\|\nabla v\|_{L^{2}\left(\Omega_{k}^{b l,-}\right)}^{2}+\|D(v)\|_{L^{2}\left(\Omega_{k}^{b l,-}\right)}^{2} .
$$

The first step relies on the notion of transparent boundary conditions in numerical analysis. The formal idea is the following: the solution $v$ of (BL) satisfies the boundary value problem

$$
\left\{\begin{aligned}
-\Delta v+\nabla q & =0, y_{2}>0, \\
\nabla \cdot v & =0, y_{2}>0, \\
\left.v\right|_{y_{2}=0} & =v_{0},
\end{aligned}\right.
$$

where $v_{0}=\left.v\right|_{y_{2}=0}$. Using the Poisson kernel for the Stokes problem in a half-plane, we have the representation formula:

$$
\begin{aligned}
& v(y)=\int_{\mathbb{R}} G\left(t, y_{2}\right) v_{0}\left(y_{1}-t\right) d t, \quad q(y)=\int_{\mathbb{R}} \nabla g\left(t, y_{2}\right) \cdot v_{0}\left(y_{1}-t\right) d t
\end{aligned}
$$

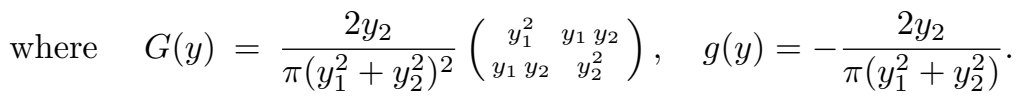

Thanks to this representation formula, we can express the stress

$$
\left.\left(\partial_{\nu} v-q \nu\right)\right|_{y_{2}=0}=-\partial_{2} v+q e_{2}
$$

in terms of $v$ at $y_{2}=0$. Formally, this leads to some relation

$$
\left.\left(-\partial_{2} v+q e_{2}\right)\right|_{\left\{y_{2}=0\right\}}=D N\left(\left.v\right|_{\left\{y_{2}=0\right\}}\right)
$$

for some Dirichlet-to-Neumann type operator $D N$. Hence, and still at a formal level, we can replace the system (BL)-(BL-Di) by the following system in $\Omega^{b l,-}$ :

$$
\left\{\begin{aligned}
&-\Delta v+\nabla q=0, y \in \Omega^{b l,-}, \\
& \nabla \cdot v=0, y \in \Omega^{b l,-}, \\
& v=-\left(y_{2}, 0\right), y \in \partial \Omega^{b l,-}, \\
&\left.\left(-\partial_{2} v+q e_{2}\right)\right|_{\left\{y_{2}=0\right\}}=D N\left(\left.v\right|_{\left\{y_{2}=0\right\}}\right) .
\end{aligned}\right.
$$

Performing energy estimates as in the previous section, in the spirit of the work by Ladyzhenskaya and Solonnikov [22], leads to the existence of a solution $v$ of (BL)-(BL-Di) in $H_{l o c}^{1}\left(\Omega^{b l}\right)$ such that

$$
\sup _{k} \int_{\Omega_{k, k+1}^{b l}}|\nabla v|^{2}<+\infty
$$

The case of the boundary condition (BL-Na) has been treated by the last two authors in [12], using arguments similar to the ones above. The main difference comes from the lack of a Poincaré or Korn type inequality in $\Omega^{b l,-}$, since the Dirichlet boundary condition is replaced by a Navier condition. Therefore an additional assumption is required in order to ensure the uniqueness and existence of $v$, namely

(H") There exists $\mathcal{C}>0$, such that for all 2-D fields $u \in C_{c}^{\infty}(\bar{R})$ satisfying $\left.u \cdot \nu\right|_{\Gamma}=0$,

$$
\|u\|_{L^{2}(R)} \leq \mathcal{C}\|D(u)\|_{L^{2}(R)}, \quad D(u)=\frac{1}{2}\left(\nabla u+(\nabla u)^{t}\right) .
$$


This assumption is related to the paper by Desvillettes and Villani [14], in which the authors prove that for all bounded domains $\Omega \subset \mathbb{R}^{N}$ which lack an axis of symmetry, there exists a constant $K(\Omega)>0$ such that

$$
\begin{gathered}
\|D(u)\|_{L^{2}(\Omega)} \geq K(\Omega)\|\nabla u\|_{L^{2}(\Omega)} \\
\forall u \in H^{1}(\Omega)^{N} \text { s.t. }\left.u \cdot \nu\right|_{\partial \Omega}=0 .
\end{gathered}
$$

As in [14], it is proved in [12] that condition ( $\left.\mathrm{H}^{\prime \prime}\right)$ expresses the absence of rotational invariance of the boundary $\Gamma$. Let us stress that this notion is related, although not equivalent, to the nondegeneracy assumption $\left(\mathrm{H}^{\prime}\right)$ : it is clear that $\left(\mathrm{H}^{\prime \prime}\right)$ implies $\left(\mathrm{H}^{\prime}\right)$. However, the converse does not seem to hold in general. In a periodic setting, both inequalities are equivalent to the condition " $\omega$ not constant".

\subsection{Definition of the slip length}

The solution of (BL) now at hand, the Ansatz (3.1) shows that we still need to understand its behaviour as $y_{2}$ goes to infinity in order to derive a wall law. In the periodic context, Fourier analysis shows that solutions of the Stokes system (3.2) converge towards constants as $y_{2} \rightarrow \infty$. Moreover, the constant is equal to

$$
\int_{\mathbb{T}} v\left(y_{1}, c\right) d y_{1}
$$

for all $c \geq 0$, and because of the divergence free condition, it can be proved that

$$
\int_{\mathbb{T}} v_{2}\left(y_{1}, 0\right) d y_{1}=0 .
$$

Additionally, the Fourier coefficients corresponding to non-zero modes in $y_{1}$ all converge towards zero asymptotically fast. Setting

we thus obtain

$$
\alpha:=\int_{\mathbb{T}} v_{1}\left(y_{1}, 0\right) d y_{1},
$$

$$
v\left(\frac{y}{\varepsilon}\right) \simeq(\alpha, 0)
$$

up to exponentially small terms, as soon as $y_{2} \gg \varepsilon$. This leads to a Navier wall law of the type (Na) with $\lambda=6 \alpha \varepsilon$, for which we will give some error estimates in the next paragraph.

In a general setting, such a convergence does not always hold: a counterexample is constructed in [17] for the Stokes system (3.2). When transposed to the original system (BL) this suggests that there may be some $\omega$ for which $v$ does not converge transversally to the boundary. For the corresponding rough channels, the Dirichlet boundary condition can certainly not be improved. Therefore, in order to retrieve a Navier wall law, an additional ergodicity property must be added. The following result is proved in [5] in a random stationary and ergodic setting:

Proposition 4. There exists $\alpha \in \mathbb{R}$ such that the solution $v$ of (BL)-(BL-Di) satisfies

$$
v(y) \rightarrow(\alpha, 0), \quad \text { as } y_{2} \rightarrow+\infty
$$

locally uniformly in $y_{1}$, almost surely and in $L^{p}(M)$ for all finite $p$.

This proposition is based on the integral representation (3.3), and the ergodic theorem. It remains true for the system (BL)-(BL-Na) (the condition at the rough boundary does not play any role beyond ensuring that the second component of the limit velocity is zero).

From this convergence result, the last two authors have derived in [12] a series of estimates on $v$, using an idea developed by Souganidis [25]. The difficulty is that the use of the ergodic theorem in Proposition 4 prevents from deriving convergence estimates in $L_{u l o c}^{2}\left(\Omega^{b l}\right)$, for instance. However, this difficulty can be bypassed by using norms of the type

$$
\sup _{R \geq 1} \frac{1}{R^{1 / 2}}\left(\int_{-R}^{R} \int_{\omega\left(y_{1}\right)}^{\infty}|v(y)-(\alpha, 0)|^{2} d y\right)^{1 / 2},
$$


which are weaker than $L_{u l o c}^{2}$.

The main estimates derived in [12] are the following:

Lemma 5. - Regularity estimate: Let $\beta \in \mathbb{N}^{2}$ be arbitrary, and let $v$ be the solution of (BL). Then for all $a>0$, there exists a constant $C$, depending only on the Lipschitz constant of $\omega$, on $\beta$ and on $a$, such that

$$
\sup _{k \in \mathbb{Z}} \int_{k}^{k+1} \int_{a}^{\infty}\left|\nabla^{\beta} \nabla v\right|^{2} \leq C .
$$

- Convergence estimates: almost surely,

$$
\begin{array}{r}
\sup _{R \geq 1} \frac{1}{R^{1 / 2}}\|v(\cdot / \varepsilon)-(\alpha, 0)\|_{L^{2}\left(\Omega_{R}\right)}=o(1), \\
\sup _{R \geq 1} \frac{1}{R^{1 / 2}}\left(\left\|\int_{0}^{x_{1}} v_{2}\left(\frac{x_{1}^{\prime}}{\varepsilon}, \frac{1}{\varepsilon}\right) d x_{1}^{\prime}\right\|_{H^{3}(-R, R)}+\left\|v_{1}\left(\frac{x_{1}}{\varepsilon}, \frac{1}{\varepsilon}\right)-\alpha\right\|_{H^{3}(-R, R)}\right)=o(1) .
\end{array}
$$

Let us also mention that it is possible to derive upper and lower bounds for the "slip length" $\alpha$. Such bounds were established by Achdou et al. [2] in the periodic setting. They are still valid in the random stationary case. Along the lines of [3], one can prove: for $\omega \in \mathcal{C}^{2}(\mathbb{R}) \cap W^{2, \infty}(\mathbb{R})$,

$$
-\sup _{\mathbb{R}} \omega \leq \alpha \leq-\inf _{\mathbb{R}} \omega
$$

\subsection{Proof of convergence}

Once the slip length is defined, there remains to derive estimates for the difference $u^{\varepsilon}-u^{N}$, where $u^{N}$ is the solution of (NS)-(Na) with $\lambda=6 \alpha \varepsilon$. The idea is to introduce an approximation of $u^{\varepsilon}$ defined as follows:

$$
u_{\mathrm{app}}^{\varepsilon}(x):=u^{0}(x)+6 \phi \varepsilon v\left(\frac{x}{\varepsilon}\right)+\varepsilon u^{1}(x)+\varepsilon r^{\varepsilon}(x)+6 \phi \varepsilon^{2} v^{1}\left(\frac{x}{\varepsilon}\right),
$$

where the correctors $u^{1}$ and $r^{\varepsilon}$ ensure that $u_{\mathrm{app}}^{\varepsilon}$ satisfies the Dirichlet boundary condition at the upper boundary and the zero flux condition. The term $v^{1}$ is a boundary layer term which compensates the tangential trace of $u^{0}+6 \phi \varepsilon v$ at the rough boundary ${ }^{1}$.

Additionally, $u^{1}$ is intended to be $O(1)$ while $r^{\varepsilon}=o(1)$.

$\triangleright$ The term $u^{1}$ is the solution of

$$
\left\{\begin{array}{l}
-\Delta u^{1}+u^{0} \cdot \nabla u^{1}+u^{1} \cdot \nabla u^{0}+\nabla p^{1}=0, \quad x \in \Omega, \\
\nabla \cdot u^{1}=0, \quad x \in \Omega, \\
u_{\mid x_{2}=0}^{1}=0, \quad u_{\mid x_{2}=1}^{1}=(-6 \phi \alpha, 0), \\
\int_{\sigma}^{1} u_{1}^{1}=-6 \phi \alpha .
\end{array}\right.
$$

Notice that we assume that $u^{1}$ satisfies a no-slip condition at the lower boundary. This stems from the non-degeneracy of the frontier $\Gamma^{\varepsilon}$ : in order that the non-penetration condition is satisfied at order $\varepsilon, u^{1}$ must vanish at $x_{2}=0$. We recall that the same argument led to the no-slip condition for $u^{0}$ at $x_{2}=0$.

Hence the vector field $u^{1}$ is a combination of Couette and Poiseuille flows:

$$
u_{1}^{1}(x)=6 \phi\left(-4 \alpha x_{2}+3 \alpha x_{2}^{2}\right), \quad u_{2}^{1}(x)=0, \quad x \in \Omega
$$

and we extend $u^{1}$ by zero outside $\Omega$.

\footnotetext{
${ }^{1}$ It can be checked that this extra boundary layer term is needed only when the original slip length $\lambda^{\varepsilon}$ is of order one.
} 
$\triangleright$ The additional boundary term $v^{1}$ solves the system

$$
\left\{\begin{array}{l}
-\Delta v^{1}+\nabla q_{1}=0 \quad \text { in } \Omega^{b l} \\
v^{1} \cdot \nu=\left(y_{2}^{2}, 0\right) \cdot \nu \quad \text { on } \Gamma, \\
\lambda^{0}\left(D\left(v^{1}\right) \nu\right)_{\tau}=\left(v+\left(y_{2}, 0\right)\right)_{\tau}+\lambda^{0}\left(D\left(\left(y_{2}^{2}, 0\right)\right) \nu\right)_{\tau} \text { on } \Gamma,
\end{array}\right.
$$

under the condition

$$
\sup _{k \in \mathbb{Z}} \int_{\Omega_{k, k+1}^{b l}}\left|\nabla v^{1}\right|^{2} .
$$

Using the same techniques as the ones briefly described above, energy estimates in $H_{u l o c}^{1}\left(\Omega_{-}^{b l}\right)$ for $v^{1}$ can be proved, leading to existence and uniqueness of $v^{1}$. Additionally, there exists $\beta \in \mathbb{R}$ such that

$$
\lim _{y_{2} \rightarrow \infty} v^{1}\left(y_{1}, y_{2}\right)=(\beta, 0)
$$

almost surely, and exponentially fast in the periodic case.

Notice that to simplify the presentation, we have chosen $\lambda^{\varepsilon} \equiv \lambda^{0}>0$.

$\triangleright$ Part of the quality of the approximation is limited by the corrector term $r^{\varepsilon}$. The idea is to choose a vector field $r^{\varepsilon} \in H_{\text {loc }}^{2}(\Omega)$ satisfying

$$
\left\{\begin{array}{l}
\nabla \cdot r^{\varepsilon}=0, \\
r_{\mid x_{2}=0}^{\varepsilon}=0, \quad r_{\mid x_{2}=1}^{\varepsilon}=6 \phi\left[(\alpha, 0)-v\left(\frac{x_{1}}{\varepsilon}, \frac{1}{\varepsilon}\right)-\varepsilon v^{1}\left(\frac{x_{1}}{\varepsilon}, \frac{1}{\varepsilon}\right)\right], \\
\int_{\sigma}^{\mid} r_{1}^{\varepsilon}=-\int_{\sigma^{\varepsilon} \backslash \sigma} u_{1}^{0}-6 \phi \int_{\sigma_{\varepsilon}}\left(v_{1}+\varepsilon v_{1}^{1}\right)(x / \varepsilon)+6 \phi \alpha,
\end{array}\right.
$$

Of course, the precise definition of $r^{\varepsilon}$ depends on the model under consideration: for instance, in [19], there is no condition on the flux of $u^{\varepsilon}$; however, the pressures on the lateral boundaries of the bounded domain are prescribed. Therefore, in that case, $r^{\varepsilon}$ rather takes the form of a boundary layer corrector located on the lateral sides of the domain.

In the periodic case, $v(y)$ converges exponentially fast towards $(\alpha, 0)$ as $y_{2} \rightarrow \infty$, so that

$$
r_{\mid x_{2}=1}^{\varepsilon}=O(\varepsilon), \quad \int_{\sigma} r_{1}^{\varepsilon}=O(\varepsilon) .
$$

Hence in this case, it is possible to find a vector field $r^{\varepsilon}$ such that $r^{\varepsilon}=O(\varepsilon)$ in $H^{2}$. In the general stationary ergodic case, however, no rate of convergence is known for the convergence of the boundary layer term. Therefore the only available estimate on $r^{\varepsilon}$ is

$$
\sup _{R \geq 1} \frac{1}{R^{1 / 2}}\left\|r^{\varepsilon}\right\|_{H^{2}\left(\Omega \cap\left\{\left|x_{1}\right| \leq R\right\}\right)}=o(1) \text { and }\left\|r^{\varepsilon}\right\|_{W^{2, \infty}(\Omega)}=O(1) .
$$

The construction of such a function $r^{\varepsilon}$ is given in [5], and the above estimates in the stationary ergodic case are derived in [12].

Let us also mention that in [16], the third author studies the case when the rough boundary has no correlation at large distances. In this case, the convergence of $v$ towards $(\alpha, 0)$ is much sharper than in the general random case, and in particular, it can be proved that

$$
\left\|r^{\varepsilon}\right\|_{H_{u l o c}^{2}(\Omega)}=O\left(\varepsilon^{1 / 2}|\ln (\varepsilon)|^{1 / 2}\right) .
$$

By construction, the function $u_{\text {app }}^{\varepsilon}$ satisfies

$$
\left\{\begin{array}{l}
-\Delta u_{\mathrm{app}}^{\varepsilon}+u_{\mathrm{app}}^{\varepsilon} \cdot \nabla u_{\mathrm{app}}^{\varepsilon}+\nabla p_{\mathrm{app}}^{\varepsilon}=\operatorname{div} G^{\varepsilon}+f^{\varepsilon} \quad \text { in } \Omega^{\varepsilon} \backslash \Sigma, \\
u_{\mathrm{app}}^{\varepsilon} \mid x_{2}=1=0, \\
\left.u_{\mathrm{app}}^{\varepsilon} \cdot \nu\right|_{\Gamma^{\varepsilon}}=0, \\
\left.\left(u_{\mathrm{app}}^{\varepsilon}\right)\right|_{\Gamma^{\varepsilon}}=\left.\lambda^{0}\left(D\left(u_{\mathrm{app}}^{\varepsilon}\right) \nu\right)_{\tau}\right|_{\Gamma^{\varepsilon}}+g^{\varepsilon}, \\
{\left.\left[u_{\mathrm{app}}^{\varepsilon}\right]\right|_{\Sigma}=0,\left.\quad\left[D\left(u_{\mathrm{app}}^{\varepsilon}\right) e_{2}-p^{\varepsilon} e_{2}\right]\right|_{\Sigma}=\left.D\left(\varepsilon r^{\varepsilon}+\varepsilon u^{1}\right) e_{2}\right|_{\Sigma}=: \varphi^{\varepsilon}}
\end{array}\right.
$$


where

$$
\begin{aligned}
G^{\varepsilon} & =\varepsilon u^{0} \otimes\left(6 \phi\left(v\left(\frac{\cdot}{\varepsilon}\right)-(\alpha, 0)+\varepsilon v^{1}\left(\frac{\cdot}{\varepsilon}\right)\right)+r^{\varepsilon}\right) \\
& +\varepsilon\left(6 \phi\left(v\left(\frac{\dot{\varepsilon}}{\varepsilon}\right)-(\alpha, 0)+\varepsilon v^{1}\left(\frac{\dot{\varepsilon}}{\varepsilon}\right)\right)+r^{\varepsilon}\right) \otimes u^{0} \\
& +\varepsilon^{2}\left(6 \phi\left(v\left(\frac{\cdot}{\varepsilon}\right)+\varepsilon v^{1}\left(\frac{\cdot}{\varepsilon}\right)\right)+r^{\varepsilon}\right) \otimes\left(6 \phi\left(v\left(\frac{\cdot}{\varepsilon}\right)+\varepsilon v^{1}\left(\frac{\cdot}{\varepsilon}\right)\right)+r^{\varepsilon}\right)
\end{aligned}
$$

and

$$
f^{\varepsilon}=-\varepsilon \Delta r^{\varepsilon}, \quad g^{\varepsilon}=\left.6 \phi\left(\varepsilon^{2} v^{1}(x / \varepsilon)-\left(x_{2}^{2}, 0\right)\right)_{\tau}\right|_{\Gamma^{\varepsilon}} .
$$

The error terms satisfy estimates of the type

$$
\left\|G^{\varepsilon}\right\|=o(\varepsilon), \quad\left\|f^{\varepsilon}\right\|=o(\varepsilon), \quad\left\|\varphi^{\varepsilon}\right\|=o(\varepsilon)+O(\varepsilon \phi), \quad\left\|g^{\varepsilon}\right\|=O\left(\varepsilon^{2}\right)
$$

in adequate norms (we refer to [12] for more details). Setting $w^{\varepsilon}=u^{\varepsilon}-u_{\mathrm{app}}^{\varepsilon}$, we obtain

$$
-\Delta w^{\varepsilon}+\left(u^{\varepsilon} \cdot \nabla\right) w^{\varepsilon}+\left(w^{\varepsilon} \cdot \nabla\right) u_{\mathrm{app}}^{\varepsilon}+\nabla q^{\varepsilon}=-\operatorname{div} G^{\varepsilon}-f^{\varepsilon} \quad \text { in } \Omega^{\varepsilon} \backslash \Sigma,
$$

and $w^{\varepsilon}$ satisfies the same boundary and jump conditions as $u_{\text {app }}^{\varepsilon}$.

Writing energy estimates on $w^{\varepsilon}$ in the same spirit as in Section 2 leads to

$$
\sup _{R \geq 1} \frac{1}{R^{1 / 2}}\left\|u^{\varepsilon}-u_{\mathrm{app}}^{\varepsilon}\right\|_{L^{2}\left(\Omega^{\varepsilon} \cap\left\{\left|x_{1}\right| \leq R\right\}\right.}=o(\varepsilon) \quad \text { almost surely. }
$$

In the periodic case, this estimate becomes (see [19])

$$
\left\|u^{\varepsilon}-u_{\mathrm{app}}^{\varepsilon}\right\|_{L_{u l o c}^{2}}=O\left(\varepsilon^{3 / 2}\right) .
$$

On the other hand, the function $u^{N}$ is explicit, namely

$$
u^{N}=\left(6 \phi U^{N}\left(x_{2}\right), 0\right) \quad \text { with } U_{N}\left(x_{2}\right)=-\frac{1+\varepsilon \alpha}{1+4 \varepsilon \alpha} x_{2}^{2}+\frac{1}{1+4 \varepsilon \alpha} x_{2}+\frac{\varepsilon \alpha}{1+4 \varepsilon \alpha},
$$

so that

$$
u^{N}=u^{0}+6 \phi \varepsilon(\alpha, 0)+\varepsilon u^{1}+O\left(\varepsilon^{2}\right) \text { in } L_{u l o c}^{2}(\Omega) .
$$

From there, we obtain

$$
\sup _{R \geq 1} \frac{1}{R^{1 / 2}}\left\|u^{N}-u^{\varepsilon}\right\|_{L^{2}\left(\Omega_{R}^{\varepsilon}\right)}=o(\varepsilon) \quad \text { almost surely }
$$

in the random stationary case, and $\left\|u^{\varepsilon}-u^{N}\right\|_{L_{u l o c}^{2}}=O\left(\varepsilon^{3 / 2}\right)$ in the periodic case. In the random case with no correlation at large distance [16], the estimate becomes $\left\|u^{\varepsilon}-u^{N}\right\|_{L_{u l o c}^{2}}=$ $O\left(\varepsilon^{3 / 2}|\ln (\varepsilon)|^{1 / 2}\right)$.

\section{Optimizing THE SLIP IN A PERIODIC SETTING}

This section is concerned with the optimization of the slip length $\alpha$, starting from the boundary layer system (BL)-(BL-Di). We prove that for adherent boundaries, i.e. boundaries on which the fluid satisfies a no-slip condition $\left(\lambda^{\varepsilon}=0\right)$, the roughness does not enhance slip. Our result holds in two-dimensional and three-dimensional settings. In three dimensions, we merely replace (BL-Di) by

$$
\left.v\right|_{\Gamma}=-y_{d}\left(e_{h}, 0\right),
$$

for some $e_{h} \in \mathbb{R}^{d-1},\left|e_{h}\right|=1$; notice that if we take $d=2$ in (4.1), we retrieve (BL-Di). 
- We start by making precise the setting of our study. Let $\omega: \mathbb{T}^{d-1} \rightarrow \mathbb{R}$ be a continuous function $(d=2$ or 3$)$, and let

$$
\Omega_{\omega}^{b l}:=\left\{\left(y_{h}, y_{d}\right) \in \mathbb{T}^{d-1} \times \mathbb{R}, y_{d}>\omega\left(y_{h}\right)\right\} .
$$

When $\lambda^{\varepsilon}=0$, the boundary layer system is (BL)-(4.1). It has a unique solution $v \in H_{l o c}^{1}\left(\Omega_{\omega}^{b l}\right)$ such that

$$
\int_{\Omega_{\omega}^{b l}}|\nabla v|^{2}<\infty
$$

which we denote in the rest of this section by $v_{\omega}\left(\cdot ; e_{h}\right)$.

It can be checked that there exists a matrix $M=M(\omega) \in \mathcal{M}_{d-1}(\mathbb{R})$ such that

$$
M e_{h}=\lim _{y_{d} \rightarrow \infty} v_{\omega, h}\left(y_{h}, y_{d} ; e_{h}\right)=\left\langle v_{\omega, h}\left(\cdot, c ; e_{h}\right)\right\rangle,
$$

where $c$ is an arbitrary constant chosen so that $c>\sup \omega$. The homogenized Navier condition at the lower boundary is then

$$
\left.u_{h}^{\varepsilon}\right|_{x_{d}=0}=\left.\varepsilon M \partial_{d} u_{h}^{\varepsilon}\right|_{x_{d}=0} .
$$

The slip length, which we wish to optimize, is defined as

$$
\alpha(\omega):=\sup _{e_{h} \in \mathbb{R}^{d-1},\left|e_{h}\right|=1}\left\langle M e_{h}, e_{h}\right\rangle .
$$

The goal of the present section is to maximize $\alpha(\omega)$ among all roughness profiles $\omega$. However this optimization only makes sense provided the average of $\omega$ is prescribed: indeed, it can be easily seen on system (BL)-(4.1) that for all $H \in \mathbb{R}, y \in \Omega_{\omega+H}^{b l}$,

$$
v_{\omega+H}\left(y_{h}, y_{d} ; e_{h}\right)=v_{\omega}\left(y_{h}, y_{d}-H ; e_{h}\right)-H\left(e_{h}, 0\right) .
$$

Hence $M(\omega+H)=M(\omega)-H$ Id and therefore $\alpha(\omega+H)=\alpha(\omega)-H$ for all $H \in \mathbb{R}$ : translating the rough surface results in a translation of the slip coefficient. Therefore, instead of maximizing $\alpha$, we maximize

$$
\tilde{\alpha}(\omega):=\alpha(\omega)+\langle\omega\rangle
$$

this amounts to prescribing the average height of the rough surface. In terms of the original system $\left(\mathrm{NS}^{\varepsilon}\right)$, this is equivalent to working with a given volume for the channel $\Omega^{\varepsilon}$.

Our result is the following:

Theorem 6. - The supremum problem is well-defined:

$$
\sup _{\omega \in \mathcal{C}\left(\mathbb{T}^{2}\right)} \tilde{\alpha}(\omega)<+\infty .
$$

- Maximum slip coefficient is achieved for flat surfaces:

$$
\max _{\omega \in \mathcal{C}\left(\mathbb{T}^{2}\right)} \tilde{\alpha}(\omega)=\tilde{\alpha}(0)=0 .
$$

Remark 7. (i) This theorem means that the roughness does not enhance slip for adherent surfaces. In other words, the Navier wall law should really be seen as a means to effectively compute the velocity of the fluid, but not as the expression of an increase of the surface slip.

(ii) The above theorem implies in particular

$$
\alpha \leq-\langle\omega\rangle,
$$

which is more precise than the previous estimate (3.5) (see [3]). 
Proof. We give two different proofs of the Theorem. The first one is very short, and relies merely on a Poincaré inequality. The second is slightly longer, but more general, and could probably be adapted to other contexts. Therefore we have chosen to include it in the present paper.

- Reformulation of (BL)-(4.1) and associated energy.

First, notice that thanks to the invariance by translation of the function $\tilde{\alpha}$, we can always assume that we work with functions $\omega$ such that $\sup \omega \leq-1 / 2$, for instance, so that the rough boundary does not intersect the surface $y_{d}=0$.

In order to reduce the problem to a scalar equation, it is easier to work with homogeneous boundary conditions. Hence we define

$$
u_{\omega}\left(y ; e_{h}\right)=v_{\omega}\left(y ; e_{h}\right)+y_{d}\left(e_{h}, 0\right) \mathbf{1}_{y_{d}<0} .
$$

The function $u_{\omega}$ satisfies

$$
\left\{\begin{array}{c}
-\Delta u_{\omega}+\nabla p=0, \quad y \in \Omega_{\omega}^{b l} \backslash \Sigma, \\
\operatorname{div} u_{\omega}=0, \quad y \in \Omega_{\omega}^{b l}, \\
\left.u_{\omega}\right|_{\Gamma}=0 \\
{\left.\left[u_{\omega}\right]\right|_{\Sigma}=0} \\
{\left.\left[\partial_{y_{d}} u_{\omega}-p e_{d}\right]\right|_{\Sigma}=-\left(e_{h}, 0\right)}
\end{array}\right.
$$

where $\Sigma=\mathbb{T}^{d-1} \times\{0\}$ and $\left.[f]\right|_{\Sigma}=f\left(y_{h}, 0^{+}\right)-f\left(y_{h}, 0^{-}\right)$. The above system is the Euler-Lagrange equation associated with the energy

$$
\begin{array}{r}
E\left(u ; e_{h}\right)=\frac{1}{2} \int_{\Omega_{\omega}^{b l}}|\nabla u|^{2}-\int_{\Sigma} u_{h} \cdot e_{h}, \\
u \in \mathcal{H}_{\omega}:=\left\{u \in \dot{H}^{1}\left(\Omega_{\omega}^{b l}\right)^{d},\left.u\right|_{\Gamma}=0, \operatorname{div} u=0\right\} .
\end{array}
$$

On the other hand,

$$
\begin{aligned}
\left\langle M(\omega) e_{h}, e_{h}\right\rangle & =\left\langle\left. v_{\omega, h}\right|_{\Sigma} \cdot e_{h}\right\rangle=\left\langle\left. u_{\omega, h}\right|_{\Sigma} \cdot e_{h}\right\rangle \\
& =\frac{1}{\left|\mathbb{T}^{d-1}\right|} \int_{\Omega_{\omega}^{b l}}\left|\nabla u_{\omega}\right|^{2}=-\frac{2}{\left|\mathbb{T}^{d-1}\right|} E\left(u_{\omega} ; e_{h}\right) \\
& =-\frac{2}{\left|\mathbb{T}^{d-1}\right|} \min _{u \in \mathcal{H}_{\omega}} E\left(u ; e_{h}\right) .
\end{aligned}
$$

These identities prove that the supremum is well-posed, and also lead to the first proof of the Theorem. Indeed, for all $y_{h} \in \mathbb{T}^{d-1}$,

$$
u_{\omega}\left(y_{h}, 0\right)=\int_{\omega\left(y_{h}\right)}^{0} \partial_{d} u_{\omega}\left(y_{h}, y_{d}\right) d y_{d}
$$

so that, using the Cauchy-Schwarz inequality,

$$
\begin{aligned}
\int_{\Sigma} u_{\omega, h} \cdot e_{h} & =\int_{\mathbb{T}^{d-1}} \int_{\omega\left(y_{h}\right)}^{0} \partial_{d} u_{\omega, h}\left(y_{h}, y_{d}\right) \cdot e_{h} d y \\
& \leq\left(\int_{\mathbb{T}^{d-1}}\left|\omega\left(y_{h}\right)\right| d y_{h}\right)^{1 / 2}\left(\int_{\mathbb{T}^{d-1}} \int_{\omega\left(y_{h}\right)}^{0}\left|\partial_{d} u_{\omega, h}\left(y_{h}, y_{d}\right) \cdot e_{h}\right|^{2} d y\right)^{1 / 2} \\
& \leq\left|\int_{\mathbb{T}^{d-1}} \omega\left(y_{h}\right) d y_{h}\right|^{1 / 2}\left(\int_{\Omega_{\omega}^{b l}}\left|\nabla u_{\omega}\right|^{2}\right)^{1 / 2} .
\end{aligned}
$$


Eventually, we infer

$$
\left\langle M(\omega) e_{h}, e_{h}\right\rangle=\left\langle\left. v_{\omega, h}\right|_{\Sigma} \cdot e_{h}\right\rangle \leq|\langle\omega\rangle|,
$$

and thus $\alpha(\omega) \leq|\langle\omega\rangle|$. Therefore

$$
\tilde{\alpha}(\omega) \leq 0 \quad \forall \omega .
$$

Since $\tilde{\alpha}(\omega)=0$ if $\omega$ is constant, the theorem is proved.

- Alternative proof based on the study of a scalar problem:

In order to simplify the presentation, we assume from now on, without loss of generality, that $\left|\mathbb{T}^{d-1}\right|=1$. The idea is the following: since $v_{\omega}$ is scalar when $\omega$ is constant, we study the optimization for a scalar problem associated with (BL)-(4.1), and we prove that the optimum in the scalar case is attained by a flat boundary.

First, we deduce from the equalities of the previous paragraph that for all $e_{h} \in \mathbb{R}^{d-1}$ such that $\left|e_{h}\right|=1$,

$$
\sup _{\omega \in \mathcal{C}\left(\mathbb{T}^{d-1}\right)}\left(\left\langle M(\omega) e_{h}, e_{h}\right\rangle+\langle\omega\rangle\right)=-2 \inf _{\omega \in \mathcal{C}\left(\mathbb{T}^{d-1}\right)}\left(\min _{u \in \mathcal{H}_{\omega}} E\left(u ; e_{h}\right)-\frac{1}{2}\langle\omega\rangle\right) .
$$

Obviously, for all $u=\left(u_{1}, \cdots, u_{d}\right) \in \mathcal{H}_{\omega}$,

$$
\int_{\Omega_{\omega}^{b l}}|\nabla u|^{2} \geq \int_{\Omega_{\omega}^{b l}}\left|\nabla\left(u \cdot e_{h}\right)\right|^{2}
$$

so that

$$
\min _{u \in \mathcal{H}_{\omega}} E(u) \geq \min _{\substack{\left.u \in \dot{H}^{1}\left(\Omega_{\omega}^{b l}\right)^{d} \\ u\right|_{\Gamma}=0}}\left(\frac{1}{2} \int_{\Omega_{\omega}^{b l}}\left|\nabla u \cdot e_{h}\right|^{2}-\int_{\Sigma} u \cdot e_{h}\right) .
$$

Hence we now consider the minimization problem

$$
I_{1}:=\inf _{\omega \in \mathcal{C}\left(\mathbb{T}^{d-1}\right)} \min _{\substack{g \in \dot{H}^{1}\left(\Omega_{\omega}^{b l}\right),\left.g\right|_{\Gamma}=0}}\left(\frac{1}{2} \int_{\Omega_{\omega}^{b l}}|\nabla g|^{2}-\int_{\Sigma} g-\frac{1}{2}\langle\omega\rangle\right) .
$$

Notice that the function $g$ above is scalar.

For $y_{h} \in \mathbb{T}^{d-1}, g \in \dot{H}^{1}\left(\Omega_{\omega}^{b l}\right)$, define

$$
F\left[g ; y_{h}\right]:=\frac{1}{2} \int_{\omega\left(y_{h}\right)}^{\infty}\left|\nabla g\left(y_{h}, y_{d}\right)\right|^{2} d y_{d}-\left.g\right|_{y_{d}=0}\left(y_{h}\right)-\frac{1}{2} \omega\left(y_{h}\right) .
$$

By definition,

$$
I_{1}=\inf _{\omega \in \mathcal{C}\left(\mathbb{T}^{d-1}\right)} \min _{\substack{\left.g \in \dot{H}^{1}\left(\Omega_{\omega}^{b l}\right) \\ g\right|_{\Gamma}=0}} \int_{\mathbb{T}^{d-1}} F\left[g ; y_{h}\right] d y_{h} .
$$

For all $g \in \dot{H}^{1}\left(\Omega_{\omega}^{b l}\right)$ such that $\left.g\right|_{\Gamma}=0$, there exists $\bar{y}_{h} \in \mathbb{T}^{d-1}$ such that

$$
F\left[g ; \bar{y}_{h}\right] \leq \int_{\mathbb{T}^{d-1}} F\left[g ; y_{h}\right] d y_{h} .
$$

Let

$$
\begin{array}{r}
\bar{g}\left(y_{h}, y_{d}\right)=g\left(\bar{y}_{h}, y_{d}\right), \\
\bar{\omega}\left(y_{h}\right)=\omega\left(\bar{y}_{h}\right) .
\end{array}
$$


Then $\bar{g} \in \dot{H}^{1}\left(\Omega_{\bar{\omega}}^{b l}\right)$, and $\left.\bar{g}\right|_{y_{d}=\bar{\omega}\left(y_{h}\right)}=g\left(\bar{y}_{h}, \omega\left(\bar{y}_{h}\right)\right)=0$. Using the definition of $\bar{y}_{h}$ together with the fact that $\bar{g}$ does not depend on $y_{h}$, we infer

$$
\begin{aligned}
& \frac{1}{2} \int_{\Omega_{\omega}^{b l}}|\nabla g|^{2}-\int_{\Sigma} g-\frac{1}{2}\langle\omega\rangle=\int_{\mathbb{T}^{d-1}} F\left[g, y_{h}\right] d y_{h} \\
\geq & \frac{1}{2} \int_{\omega\left(\bar{y}_{h}\right)}^{\infty}\left|\nabla g\left(\bar{y}_{h}, y_{d}\right)\right|^{2}-g\left(\bar{y}_{h}, 0\right)-\frac{1}{2} \omega\left(\bar{y}_{h}\right) \\
\geq & \frac{1}{2} \int_{\bar{\omega}\left(y_{h}\right)}^{\infty}\left|\nabla \bar{g}\left(y_{h}, y_{d}\right)\right|^{2}-\bar{g}\left(y_{h}, 0\right)-\frac{1}{2} \bar{\omega}\left(y_{h}\right) \\
= & \frac{1}{2} \int_{\Omega_{\omega}^{b l}}|\nabla \bar{g}|^{2}-\int_{\Sigma} \bar{g}-\frac{1}{2}\langle\bar{\omega}\rangle .
\end{aligned}
$$

Hence the minimum in $I_{1}$ is attained for constant $\omega$ and for $g$ which does not depend on $y_{h}$ :

$$
I_{1}=\inf _{H<0} \min _{\substack{g \in \dot{H}^{1}(H, \infty),\left.g\right|_{y_{d}=H}=0}}\left(\frac{1}{2} \int_{H}^{\infty}\left|\partial_{d} g\right|^{2}-\left.g\right|_{y_{d}=0}-\frac{H}{2}\right) .
$$

In conclusion, we have proved that

$$
\inf _{H<0} \min _{\substack{\left.g \in \dot{H}^{1}(H, \infty) \\ g\right|_{y_{d}}=H=0}}\left(\frac{1}{2} \int_{H}^{\infty}\left|\partial_{d} g\right|^{2}-\left.g\right|_{y_{d}=0}-\frac{H}{2}\right) \leq \inf _{\omega \in \mathcal{C}\left(\mathbb{T}^{d-1}\right)}\left(\min _{u \in \mathcal{H}_{\omega}} E\left(u ; e_{h}\right)-\frac{1}{2}\langle\omega\rangle\right) .
$$

On the other hand, for $H<0$ and $g \in \dot{H}^{1}(H, \infty)$ such that $\left.g\right|_{y_{d}=H}=0$, let $u=g\left(e_{h}, 0\right)$. Then $u \in \mathcal{H}_{H}$ and

Hence

$$
E\left(u ; e_{h}\right)-\frac{1}{2}\langle\omega\rangle=\frac{1}{2} \int_{H}^{\infty}\left|\partial_{d} g\right|^{2}-\left.g\right|_{y_{d}=0}-\frac{H}{2} .
$$

$$
\inf _{H<0} \min _{\substack{\left.g \in \dot{H}^{1}(H, \infty) \\ g\right|_{y_{d}}=H=0}}\left(\frac{1}{2} \int_{H}^{\infty}\left|\partial_{d} g\right|^{2}-\left.g\right|_{y_{d}=0}-\frac{H}{2}\right) \geq \inf _{\omega \in \mathcal{C}\left(\mathbb{T}^{d-1}\right)}\left(\min _{u \in \mathcal{H}_{\omega}} E\left(u ; e_{h}\right)-\frac{1}{2}\langle\omega\rangle\right) .
$$

Eventually, we deduce that

$$
\begin{aligned}
& \sup _{\omega \in \mathcal{C}\left(\mathbb{T}^{d-1}\right)} \tilde{\alpha}(\omega)=-2 \inf _{H<0} \min _{\substack{g \in \dot{H}^{1}(H, \infty),\left.g\right|_{y_{d}=H}=0}}\left(\frac{1}{2} \int_{H}^{\infty}\left|\partial_{d} g\right|^{2}-\left.g\right|_{y_{d}=0}-\frac{H}{2}\right) \\
& =0=\tilde{\alpha}(0) \text {. }
\end{aligned}
$$

\section{ACKNOWLEDGEMENTS}

Anne-Laure Dalibard and David Gérard-Varet acknowledge the support of the ANR project ANR-08-JCJC-0104-01 and Dorin Bucur the support of the ANR project ANR-09-BLAN-0037.

\section{REFERENCES}

[1] Achdou, Y., Mohammadi, B., Pironneau, O., and Valentin, F. Domain decomposition \& wall laws. In Recent developments in domain decomposition methods and flow problems (Kyoto, 1996; Anacapri, 1996), vol. 11 of GAKUTO Internat. Ser. Math. Sci. Appl. Gakkōtosho, Tokyo, 1998, pp. 1-14.

[2] Achdou, Y., Le Tallec, P., Valentin, F., and Pironneau, O. Constructing wall laws with domain decomposition or asymptotic expansion techniques. Comput. Methods Appl. Mech. Engrg. 151, 1-2 (1998), 215-232. Symposium on Advances in Computational Mechanics, Vol. 3 (Austin, TX, 1997). 
[3] Achdou, Y., Pironneau, O., and Valentin, F. Effective boundary conditions for laminar flows over periodic rough boundaries. J. Comput. Phys. 147, 1 (1998), 187-218.

[4] Amirat, Y., Bresch, D., Lemoine, J., And Simon, J. Effect of rugosity on a flow governed by stationary Navier-Stokes equations. Quart. Appl. Math. 59, 4 (2001), 769-785.

[5] Basson, A., and Gérard-Varet, D. Wall laws for fluid flows at a boundary with random roughness. Comm. Pure Applied Math. Volume 61, Issue 7, Date: July 2008, Pages: 941-987.

[6] Bonnivard, M. and Bucur, D. The Uniform Rugosity Effect. J. Math. Fluid Mech. 14 (2012), no. 2, 201-215.

[7] Bourgeat, A., Mikelić, A. And Wright, S. Stochastic two-scale convergence in the mean and applications. J. Reine Angew. Math. 456 (1994), 19-51.

[8] Bucur, D., Feireisl, E., Necasova, S., and Wolf, J. On the asymptotic limit of the Navier-Stokes system with rough boundaries J. Diff. Equations, 244 (2008), 2890-2908.

[9] Bucur, D., Feireisl, E., And Necasova, S. On the asymptotic limit of flows past a ribbed boundary. $J$. Math. Fluid Mech. 10 (2008), no. 4, 554-568.

[10] Bucur, D., Feireisl, E., And Nechsova, S. Influence of wall roughness on the slip behaviour of viscous fluids. (English summary) Proc. Roy. Soc. Edinburgh Sect. A 138 (2008), no. 5, 957-973.

[11] Casado-Diaz, J., Fernandez-Cara, E., and Simon, J. Why viscous fluids adhere to rugose walls: a mathematical explanation. J. Differential Equations 189 (2003), no. 2, 526-537.

[12] Dalibard, A.L. And Gerard-Varet, D. Effective boundary condition at a rough surface starting from a slip condition J. Differential Equations 251 (2011), no. 12, 3450-3487.

[13] Dalibard, A.L. And Saint-Raymond, L. Mathematical study of degenerate boundary layers. preprint hal00682477, arXiv:1203.5663 (2012).

[14] Desvillettes, L. And Villani, C. On a Variant of Korn's Inequality Arising in Statistical Mechanics. ESAIM, Control, Optimization and Calculus of Variations 8, (2002), 603-619, (special issue).

[15] Duvaut, G. and Lions, J. L. Les Inéquations en Mécanique et en Physique. Dunod, Paris, 1972.

[16] Gerard-Varet, D. The Navier wall law at a boundary with random roughness Comm. Math. Phys., 286, 2009, 81-110.

[17] Gerard-Varet, D., And Masmoudi, N. Relevance of the slip condition for fluid flows near an irregular boundary. Comm. Math. Phys., 295, 2010, 99-137.

[18] Gerard-Varet, D., And Masmoudi, N. Homogenization and boundary layer. To appear in Acta. Math.

[19] JäGER, W., AND Mikelić, A. On the roughness-induced effective boundary conditions for an incompressible viscous flow. J. Differential Equations 170, 1 (2001), 96-122.

[20] Jäger, W., AND Mikelić, A. Couette flows over a rough boundary and drag reduction. Comm. Math. Phys. 232, 3 (2003), 429-455.

[21] Jikov, V. V., Kozlov, S. M., And OleǏnik, O. A. Homogenization of differential operators and integral functionals. Springer-Verlag, Berlin, 1994. Translated from the Russian by G. A. Yosifian [G. A. Iosif'yan].

[22] Ladyženskaja, O. A., and Solonnikov,V. A. Determination of solutions of boundary value problems for stationary Stokes and Navier-Stokes equations having an unbounded Dirichlet integral. J. Soviet Math., 21:728761,1983

[23] Lauga, E., Brenner, M.P., and Stone, H.A. Microfluidics: The no-slip boundary condition Handbook of Experimental Fluid Dynamics,C. Tropea, A. Yarin, J. F. Foss (Eds.), Springer, 2007.

[24] Nitsche, J. A. On Korn's second inequality. R.A.I.R.O. Analyse numérique/Numerical Analysis 15 (1981), $237-348$.

[25] Souganidis, P. Cours du Collège de France, 2009.

[26] Ybert C., Barentin C., Cottin-Bizonne C., Joseph P., and Bocquet L. Achieving large slip with superhydrophobic surfaces: Scaling laws for generic geometries Physics of fluids 19, 123601 (2007). 